\title{
LIFTING SURGERIES TO BRANCHED COVERING SPACES
}

\author{
BY
}

HUGH M. HILDEN AND JOSE MARIA MONTESINOS

\begin{abstract}
It is proved that if $M^{n}$ is a branched covering of a sphere, branched over a manifold, so is $M^{n} \times S^{m}$, but the number of sheets is one more. In particular, the $n$-dimensional torus is an $n$-fold simple covering of $S^{n}$ branched over an orientable manifold. The proof involves the development of a new technique to perform equivariant handle addition. Other consequences of this technique are given.
\end{abstract}

1. Introduction. The techniques used in [5] to raise surgeries to three-dimensional branched covering spaces and the corresponding cobordism versions of those ([6] and [1]) suggest an immediate generalization to high dimensions. Thus we begin by studying the problem of adding handles equivariantly to a branched covering space. We show that we can perform equivariant surgeries in spheres of codimension $>2$ under two restrictions. First, the sphere must be "symmetric". (This can be accomplished when the sphere has dimension 1 ([6] and [1]), but it seems to be difficult, if not impossible, to establish it in general.) The second restriction is that, in some dimensions, not all of the surgeries can be lifted even if the sphere is symmetric. This is an "equivariant framing" problem (see the appendix).

The first restriction can be partially removed if we increase by one the number of sheets of the covering. In fact, doing this, we show that we can attach handles equivariantly along some "nonsymmetric" spheres of codimension $>2$. The restriction now is that the projection of the sphere must be an embedded, unknotted sphere disjoint from the branching set. By this new technique we can already solve certain problems. For example, in response to a question posed by Edmonds and Bernstein, if $M^{n}$ is a branched covering of a sphere, branched over a manifold, so is $M^{n} \times S^{m}$, but the number of sheets is one more. In particular, the four-dimensional torus is a 4-fold branched covering of $S^{4}$, branched over an orientable 2-manifold. (Note that it cannot be 3-fold by [3].) Other consequences involve representations of open-books and mapping tori as branched covering spaces.

In a second part of this paper we will study the problem of attaching handles along "nonsymmetric" spheres of codimension 2 when the projection is knotted.

The authors would like to express their appreciation to the referee for helpful observations.

Received by the editors August 10, 1978 and, in revised form, February 21, 1979.

AMS (MOS) subject classifications (1970). Primary 57A15, 57A10; Secondary 57C45.

Key words and phrases. $n$-manifolds, $n$-fold simple branched covering spaces, knots, equivariant surgery, moves. 
2. Conventions and notation. Denote by $I^{a}$ the product $[-1,1]^{a}$, by $I$ the closed interval $[0,1]$, and by $\{0\}$ the point $(0,0, \ldots, 0) \in I^{a}$. Let $D^{r}$ or $E^{r}$ be the unit ball in $\mathbf{R}^{r}$. Denote by $S^{n-1}$ the boundary of $D^{n}$. We consider $D^{n}$ included in $D^{n+1}$ under the identification of $\mathbf{R}^{n+1}$ with $\mathbf{R}^{n} \times \mathbf{R}$. Thus $D^{n}$ separates $D^{n+1}$ into two balls $D_{+}^{n}, D_{-}^{n}$, where $D_{+}^{n}$ contains $(0, \ldots, 1)$.

Except for the appendix, we work throughout in the PL category. Thus all spaces and maps will be assumed PL without it being explicitly stated. Moreover all "moves" and alterations of maps and spaces will occur at places where the branch set is a locally flat manifold. A manifold $X^{k}$ in a manifold $Y^{m}$ is locally flat if every point $x \in X$ has a closed neighborhood $U$ in $Y$ such that $(U, U \cap X)$ is PL homeomorphic to the standard ball pair $\left(D^{m} ; D^{k}\right)$.

We use the notation $p: M^{n} \stackrel{(h)}{\rightarrow}\left(N^{n}, B^{n-2}\right)$ for an $h$ to one-branched covering space with branch set $B^{n-2} \subset N^{n}$, where $N^{n}$ is a closed, orientable manifold. We call a point $x \in B^{n-2}$ simple if $p^{-1}(x)$ contains $h-1$ points and we call the branched covering $p$ simple if every point of $B^{n-2}$ is simple. Such simple branched coverings correspond to representations from $\pi_{1}\left(N^{n}-B^{n-2}\right)$ into the symmetric group of $h$ letters in which meridians are represented by transpositions.

We use the notation $M^{n} \times I^{1} \cup_{\psi} H^{k}$ to represent the result of adding a $k$-handle to $M^{n} \times I^{1}$ along $M^{n} \times\{1\}$ using some embedding $\psi: S^{k-1} \times D^{n-k+1}$ $\rightarrow M^{n}$. We use $\chi\left(M^{n}, \psi\right)$ to indicate the manifold resulting from surgery in $M^{n}$ corresponding to $\psi$. We say this surgery is type $(k, n-k+1)$ surgery.

3. The standard branched covering of a handle. There is a standard 2-fold covering $D^{n+2} \rightarrow D^{n+2}$ branched over the standard $D^{n}$. It can be constructed, for instance, by cutting open $D^{n+2}$ along the closure of one of the components of $D^{n+1}-D^{n}$, taking two copies of the resulting $(n+2)$-ball and pasting them together in the obvious way. The restriction of this covering to $S^{n+1}$ gives the standard 2-fold covering $S^{n+1} \rightarrow S^{n+1}$ branched over the standard $S^{n-1}$. Both coverings are characterized by having an unknotted branch set.

Given integers $a, b \geqslant 0, a+b=n$, there is also a standard branched covering $S^{a} \times D^{b+1} \rightarrow D^{n+1}$, branched over an embedded $S^{a-1} \times D^{b}$. Again, it is uniquely characterized by having a standard (i.e. "unknotted") branched set. It is constructed, for example as follows:

Let $D^{a} \subset S^{n+1}$ be a disc such that $D^{a} \cap S^{n-1}=S^{a-1}$. Let $N$ be a regular neighborhood of $D^{a}$, which restricts to a regular neighborhood of $S^{a-1}$ in $S^{n-1}$. Then $N$ is homeomorphic to $D^{n+1}$ and the inverse image of $N$ under the standard 2-fold covering $S^{n+1} \rightarrow S^{n+1}$ branched over $S^{n-1}$ is a regular neighborhood of $D^{a} \cup D^{a}=S^{a}$ and is, therefore, homeomorphic to $S^{a} \times D^{b+1}$. The branching set is $N \cap S^{n-1}$, which is a regular neighborhood of $S^{a-1}$ in $S^{n-1}$, and is, therefore, homeomorphic to $S^{a-1} \times D^{b}$.

Take an unknotted $n$-disc $E^{a} \times E^{b}$, properly embedded in $D^{n+2}$, which intersects $D_{-}^{n+1}$ and $D_{+}^{n+1}$ in $\partial E^{a} \times E^{b}$ and $E^{a} \times \partial E^{b}$, respectively. Thus we have a 2-fold branched covering $Q: D^{n+2} \stackrel{(2)}{\rightarrow}\left(D^{n+2}, E^{a} \times E^{b}\right)$ which is equivalent to the standard one. The restrictions of $Q$ to the inverse images of $D_{-}^{n+1}$ and $D_{+}^{n+1}$, define 
coverings

$$
\begin{aligned}
& q_{1}: S^{a} \times D^{b+1} \stackrel{(2)}{\rightarrow}\left(D_{-}^{n+1}, \partial E^{a} \times E^{b}\right), \\
& q_{2}: D^{a+1} \times S^{b} \stackrel{(2)}{\rightarrow}\left(D_{+}^{n+1}, E^{a} \times \partial E^{b}\right)
\end{aligned}
$$

which are equivalent to the standard coverings defined above.

The following lemma is now clear.

LEMMA 1. Given integers $a, b \geqslant 0, a+b=n$, there is a proper map

$$
\left(Q, q_{1}, q_{2}\right):\left(D^{a+1} \times D^{b+1}, S^{a} \times D^{b+1}, D^{a+1} \times S^{b}\right) \rightarrow\left(D^{n+2}, D_{-}^{n+1}, D_{+}^{n+1}\right)
$$

such that $Q, q_{1}, q_{2}$ are double branched coverings. The map $Q$ is the standard double branched covering of the ball, branched over a locally flat codimension two ball $E^{a} \times E^{b}$ properly embedded in $D^{n+2}$. It intersects $D_{-}^{n+1}$ and $D_{+}^{n+1}$ in $\partial E^{a} \times E^{b}$ and $E^{a} \times \partial E^{b}$, respectively, where these sets are the branching sets for the standard coverings $q_{1}$ and $q_{2}$, respectively.

Let $p: S^{a} \times D^{b+1} \rightarrow N \subset S^{n+1}$ be the standard 2-fold branched covering defined above, where $N$ is a regular neighborhood of $D^{a}$, which restricts to a regular neighborhood of $S^{a-1}$ in $S^{n-1}$. Let $\alpha: I^{n+1} \rightarrow N^{\prime} \subset$ Int $N$ denote an embedding onto a regular neighborhood $N^{\prime}$ of $D^{a}$ in $N$, such that

(i) $\alpha\left(\frac{1}{2} I^{a} \times 0\right)=D^{a}$;

(ii) $N^{\prime} \cap S^{n-1}=\alpha\left(\partial \frac{1}{2} I^{a} \times I^{n-a} \times 0\right)$.

Then, $p^{-1} N^{\prime}$ is homeomorphic to $S^{a} \times D^{b+1}$. We can construct $p^{-} N^{\prime}$ by cutting $N^{\prime}$ open along $\alpha\left(\left(I^{a}-\operatorname{Int} \frac{1}{2} I^{a}\right) \times 0\right)$, taking two copies of this, and pasting them together in the obvious way. Then property (ii) of $\alpha$ implies that we have a product structure $\tilde{\alpha}: S^{a} \times D^{b+1} \rightarrow p^{-1} N^{\prime}$ such that the following diagram is commutative

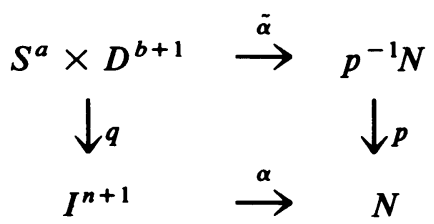

where $q$ is the standard 2 -fold branched covering. We call $\alpha$ a framing for the disc $D^{a}$, and the "symmetric" framing $\tilde{\alpha}$ for $S^{a} \times 0$ in $p^{-1} N$ is called the lifting of the framed disc $D^{a}$.

4. Connecting spheres with tubes. Let $\Sigma_{1}^{m}$ and $\Sigma_{2}^{m}$ be disjoint framed $m$-spheres in the interior of a manifold $M^{n}$. We take an embedding $\alpha: I^{n} \rightarrow M^{n}$ such that:

(i) $\alpha\left(I^{n}\right) \cap\left(\Sigma_{1}^{m} \cup \Sigma_{2}^{m}\right)=\alpha\left(\partial I^{1} \times I^{m} \times 0\right)$;

(ii) the product structure defined by $\alpha$ coincides with the framings of $\Sigma_{1}^{m}, \Sigma_{2}^{m}$, where they are simultaneously defined.

Now we define a framed sphere $\Sigma_{1,2}^{m}$ which is the connected sum, obtained by running a tube from $\Sigma_{1}^{m}$ to $\Sigma_{2}^{m}$ along $\alpha\left(I^{1} \times 0\right)$, as follows. The sphere $\Sigma_{1,2}^{m}$ is $\Sigma_{1}^{m} \cup \Sigma_{2}^{m} \cup \alpha\left(I^{1} \times \partial \frac{1}{2} I^{m} \times 0\right)-\alpha\left(\partial I^{1} \times \frac{1}{2} I^{m} \times 0\right)$. Now, one can easily define a framing for $\Sigma_{1,2}^{m}$, which coincides with the framings of $\Sigma_{1}^{m}, \Sigma_{2}^{m}$, where they are 
simultaneously defined, and also coincides with the product structure defined by $\alpha$, along the tube $\alpha\left(I^{1} \times \partial \frac{1}{2} I^{m} \times 0\right)$. This framing will be called canonical.

LEMMA 2. If $\Sigma_{2}^{m}$ is the boundary of a disc $\Delta_{2}^{m+1} \subset M^{n}$, then the sphere $\Sigma_{1,2}^{m}$ is ambient isotopic to the sphere $\Sigma_{1}^{m}$. If, in addition, the framing of $\Sigma_{2}^{m}$ extends over $\Delta_{2}^{m+1}$, then the sphere $\Sigma_{1,2}^{m}$, with canonical framing, is ambient isotopic to the framed sphere $\Sigma_{1}^{m}$.

Proof. The first part is clear: The support of the isotopy is a regular neighborhood of $\Delta_{2}^{m+1} \cup \alpha\left(I^{m+1} \times 0\right)$ in $M^{n}$. For the second assertion assume that $\beta$ : $I^{n} \rightarrow M^{n}$ extends the framing of $\Sigma_{2}^{m}$ to $\Delta_{2}^{m+1}$. Assume $\beta\left(\frac{1}{2} I^{m+1} \times 0\right)=\Delta_{2}^{m+1}$. By property (ii) of $\alpha$, we can match $\alpha$ and $\beta$ to get an embedding $\gamma: I^{n} \rightarrow M^{n}$ extending both $\alpha$ and $\beta$, and such that $\gamma\left(I^{m+1} \times 0\right)=\Delta_{2}^{m+1} \cup \alpha\left(I^{m+1} \times 0\right)$ due to property (i) of $\alpha$. We use a regular neighborhood of the image of $\gamma$ to isotope the framed $\Sigma_{1,2}^{m}$ onto the framed $\Sigma_{1}^{m}$. This proves the lemma.

5. Adding handles equivariantly along symmetric spheres. Assume we have $p$ : $M^{n} \stackrel{(h)}{\rightarrow}\left(N^{n}, B^{n-2}\right)$. Let

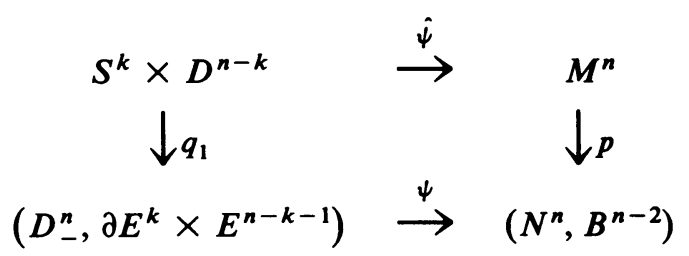

be a commutative diagram, where $0<k<n-2$ and $\psi, \hat{\psi}$ are embeddings.

Definition. $\Sigma^{k}=\hat{\psi}\left(S^{k} \times\{0\}\right)$ is called a symmetric $k$-sphere with respect to $p$, and $\hat{\psi}$ is called a symmetric framing for $\Sigma^{k}$.

In the statement of the next lemma, the reflection through $D^{n-1} \subset D^{n}$ is denoted by $\sigma_{n}$.

LEMMA 3. If (Image $\psi) \cap B^{n-2}$ consists only of simple points, then $W^{n+1}=M^{n} \times$ $I^{1} \cup_{\hat{\varphi}} H^{k+1}$ is an $h$ to one-branched covering space of $N^{n} \times I^{1}$, such that:

(i) the branching set is homeomorphic to $B^{n-2} \times I^{1} \cup_{\psi} H^{k}$;

(ii) the restriction of the covering to $M^{n} \times\{-1\}$ is $p$;

(iii) the restriction of the covering to $\partial W^{n+1}-M^{n} \times\{-1\}$ defines a covering

$$
\chi(p, \psi, \hat{\psi}): \chi\left(M^{n}, \hat{\psi}\right) \stackrel{(h)}{\rightarrow}\left(N^{n}, \chi\left(B^{n-2}, \psi\right)\right),
$$

where $\chi\left(B^{n-2}, \psi\right)$ is embedded in $N^{n}$ as

$$
\left(B^{n-2}-\psi\left(\partial E^{k} \times E^{n-k-1}\right)\right) \cup \psi \sigma_{n}\left(E^{k} \times \partial E^{n-k-1}\right) \text {; }
$$

(iv) the properties of $p$ being simple or having $B^{n-2}$ a locally flat manifold remain in $\chi(p, \psi, \hat{\psi})$.

Remark. For $k \neq 1$ the existence of $\psi$ such that (Image $\psi) \cap B^{n-2}$ is simple implies the existence of $\hat{\psi}$, making the above diagram commutative. This is not true, in general, for $k=1$. 
Proof of Lemma 3. Let $U$ and $\hat{U}$ denote the images of $\psi$ and $\hat{\psi}$, respectively. Since $U \cap B^{n-2}$ consists only of simple points, $p^{-1} U-\hat{U}$ is composed of $h-2$ components which $p$ maps homeomorphically onto $U$.

Take the disjoint union of $M^{n} \times I^{1}, h-2$ disjoint copies of $D^{n+1}$ and one copy of $D^{k+1} \times D^{n-k}$. Glue one copy of $D^{n+1}$ to each of the $h-2$ "ball" components of $p^{-1} U \times\{1\}$ along $D_{-}^{n}$, using $p^{-1} \psi$. Glue the copy of $D^{k+1} \times D^{n-k}$ to $\hat{U} \times\{1\}$ along $S^{k} \times D^{n-k}$, using $\hat{\psi}$. In this way we obtain a manifold homeomorphic to $W^{n+1}$.

Let

$$
\left(X^{n+1}, C^{n-1}\right)=\left(N^{n} \times I^{1} \cup D^{n+1}, B^{n-2} \times I^{1} \cup E^{k} \times E^{n-k-1}\right),
$$

where $\left(D^{n+1}, E^{k} \times E^{n-k-1}\right)$ is glued to $\left(N^{n}, B^{n-2}\right) \times\{1\}$ along $\left(U, U \cap B^{n-2}\right)$ $\times\{1\}$ using $\psi$.

Define $P: W^{n+1} \stackrel{(h)}{\rightarrow}\left(X^{n+1}, C^{n-1}\right)$ as follows:

$$
P=(p, 1): M^{n} \times I^{1} \rightarrow N^{n} \times I^{1} ;
$$

$P: D^{n+1} \rightarrow D^{n+1}$ is the identity in the $h-2$ "ball" copies; and $P: D^{k+1} \times D^{n-k}$ $\rightarrow D^{n+1}$ is the standard double branched covering $Q$ on the copy that is glued to $M^{n} \times\{1\}$ along $\hat{U} \times\{1\}$. A check of definitions shows that $P$ is well defined and is in fact an $h$ to one-branched covering map with branch set $C^{n-1}$ homeomorphic to $B^{n-2} \times I^{1} \cup_{\psi} H^{k}$.

The manifold $X^{n+1}$ can be identified with $N^{n} \times I^{1}$ via a homeomorphism fixed on $N^{n}-U$, since we have just glued an $(n+1)$-ball $D^{n+1}$ to $N^{n}$ along the $n$-ball $D_{-}^{n}$. This identification can be done by pushing $D^{n+1}$ down into $N^{n} \times I^{1}$ along the fibers of the natural retraction $D^{n+1} \rightarrow D^{n}$. This shows that the branching set of $P \mid \chi\left(M^{n}, \hat{\psi}\right)$ is $\left(B^{n-2}-U\right) \cup \psi \sigma_{n}\left(E^{k} \times \partial E^{n-k-1}\right)$. This proves the lemma.

The following corollary will be used in the next section.

CoROllaRY 1 ([4] OR [2]). Given $p: M^{n} \stackrel{(h)}{\rightarrow}\left(S^{n}, B^{n-2}\right)$ there exists $\tilde{p}$ : $M^{n} \stackrel{(h+1)}{\rightarrow}\left(S^{n}, \tilde{B}^{n-2}\right)$, where $\tilde{B}^{n-2}$ is the union of $B^{n-2}$ and the boundary of an $(n-1)$-ball which does not meet $B^{n-2}$.

DEFINITION. We say that $\tilde{p}$ is obtained from $p$ by addition of a trivial sheet.

Proof of Corollary 1. Consider the covering $p \cup 1: M^{n} \cup S^{n} \rightarrow\left(S^{n}, B^{n-2}\right)$ where $M^{n} \cup S^{n}$ is the disjoint union. Apply Lemma 2 for $k=0$ using an embedding $\hat{\psi}$ whose image intersects $M^{n}$ and $S^{n}$. Note that $\psi\left(D^{0} \times S^{n-2}\right)$ is the boundary of a ball which does not meet $B^{n-2}$. Identify $M^{n} \# S^{n}$ with $M^{n}$.

6. Constructing symmetric spheres. Assume we have $p: M^{n} \stackrel{(h)}{\rightarrow}\left(S^{n}, B^{n-2}\right)$ and an $m$-sphere $\Sigma^{m}$ embedded in $S^{n}$, such that $m \leqslant n-2$ and $S=p^{-1} \Sigma^{m}$ consists of $h$ spheres $S=\Sigma_{1}^{m} \cup \cdots \cup \Sigma_{h}^{m}$ (necessarily $\Sigma^{m} \cap B^{n-2}=\varnothing$; if $m \neq 1$ this is also sufficient). Let $\alpha: S^{m} \times D^{n-m} \rightarrow U$ denote a framing on a regular neighborhood $U$ of $\Sigma^{m}$. Then $\alpha$ induces framings on neighborhoods of the $\Sigma_{i}^{m}$.

Take a simple arc $a$ from $\Sigma^{m}$ to $B^{n-2}$ which intersects the branch set at one of its endpoints $P$ and the sphere $\Sigma^{m}$ only at its other endpoint $Q$. Assume that $P$ is a 
simple point. Thus, there is one, and only one, component $\tilde{a}$ of $p^{-1} a$ which connects two of the $\Sigma_{i}^{m}$, say $\Sigma_{1}^{m}$ and $\Sigma_{2}^{m}$.

There is an embedding $\beta: I^{n} \rightarrow S^{n}$ onto a regular neighborhood of $a$ such that

(i) $a=\beta([s, t] \times 0)$, for some $[s, t] \subset I^{1}$;

(ii) $\beta\left(I^{n}\right) \cap B^{n-2}=\beta\left(t \times I^{n-2} \times 0\right)$ consists of simple points;

(iii) $\beta\left(I^{n}\right) \cap \Sigma^{m}=\beta\left(s \times I^{m} \times 0\right)$;

(iv) the product structures defined by $\alpha$ and $\beta$ coincide where they are both defined (i.e. near $\beta\left(s \times I^{n-1}\right)$ ).

Let $\Delta^{m}$ be the disc $\Sigma^{m} \cup \beta\left([s, t] \times \partial \frac{1}{2} I^{m} \times 0\right)-\beta\left(s \times \frac{1}{2} I^{m} \times 0\right)$. There is now a product structure $\gamma: I^{n} \rightarrow U^{\prime}$ on a regular neighborhood of $\Delta^{m}$ in $M$, which coincides with $\alpha$ and $\beta$ where they are simultaneously defined. Moreover $\gamma\left(I^{n}\right) \cap$ $B^{n-2}$ consists of simple points. It is now easily checked that $\Delta^{m}$ is a framed disc as defined at the end of $\S 3$. We will say that the framed $\Delta^{m}$ is obtained by running $a$ tube from the framed $\Sigma^{m}$ to $B^{n-2}$ along $a$.

LEMMA 4. The lifting of the framed disc $\Delta^{m}$ is a disjoint union of $h-2$ discs and a framed symmetric $m$-sphere $\Sigma_{1,2}^{m}$ which is the result of running a tube from the framed $\Sigma_{1}^{m}$ to the framed $\Sigma_{2}^{m}$. Moreover $\Sigma_{1,2}^{m}$ has a canonical framing.

Proof. The product structure defined by the lifting of $\gamma$ is compatible with the product structures defined by the liftings of $\alpha$ and $\beta$. But it is a consequence of property (ii) of $\beta$ that the lifting of $\beta$ fulfills the conditions which serves to define a canonical framing. The rest of the proof is clear.

7. Making spheres symmetric by addition of a trivial sheet. Assume we have $p$ : $M^{n} \stackrel{(h)}{\rightarrow}\left(S^{n}, B^{n-2}\right)$ and a set $S \subset M^{n}$ composed of a number of (framed) disjoint spheres of codimension $\geqslant 2$, embedding in $M^{n}$.

Definition. We say that $X$ can be made symmetric (framed symmetric) with respect to $p$ if there is an ambient isotopy in $M^{n}$ such that each component of $S$ is mapped into a symmetric sphere with respect to $p$ (and the framings of the components of $S$ are mapped into symmetric framings with respect to $p$ ).

Let $\Sigma^{m}$ be an $m$-sphere embedded in $S^{n}$ such that

(i) $m<n-2$ and $p^{-1} \Sigma^{m}$ consists of $h m$-spheres $S=\Sigma_{1}^{m} \cup \cdots \cup \Sigma_{h}^{m}$ (necessarily $\Sigma^{m} \cap B^{n-2}=\varnothing$; if $m \neq 1$ this is also sufficient),

(ii) $\Sigma^{m}$ bounds a ball $\Delta_{0}^{m+1}$ in $S^{n}$.

We take a framing for $\Sigma^{m}$ which extends to $\Delta_{0}^{m+1}$. The framed $\Sigma^{m}$ induces framings on neighborhoods of the $\Sigma_{i}^{m}$ which we call natural.

THEOREM 1. The set $S=\Sigma_{1}^{m} \cup \cdots \cup \Sigma_{h}^{m}$, with the natural framings, can be made framed symmetric with respect to $\tilde{p}$, i.e. the result of adding a trivial sheet to $p$.

Proof. We obtain $\tilde{p}: M^{n} \stackrel{(h+1)}{\rightarrow}\left(S^{n}, B^{n-2} \cup C^{n-2}\right)$ by addition of a trivial sheet to $p$. We can assume that the ball $\Delta_{1}^{n-1}$, which $C^{n-2}$ bounds, does not meet $\Delta_{0}^{m+1} \cup B^{n-2}$. The inverse image $\tilde{p}^{-1} \Sigma^{m}$ of the framed $\Sigma^{m}$ consists of the framed spheres $\Sigma_{1}^{m}, \ldots, \Sigma_{h}^{m}$ and a new framed $m$-sphere $\Sigma_{h+1}^{m}$. Note that this sphere 
bounds a disc $\Delta_{2}^{m+1}$, which covers the disc $\Delta_{0}^{m+1}$, and that the framing of $\Sigma_{h+1}^{m}$ extends to $\Delta_{2}^{m+1}$.

Let $\tilde{C}^{n-2}$ be the component of $\tilde{p}^{-1} C^{n-2}$ consisting of the points at which $\tilde{p}$ is not a local homeomorphism. Consider one of the inverse images of $\Sigma^{m}$ by $p$, say $\Sigma_{1}^{m}$, and choose an $\operatorname{arc} \tilde{a}$ from $\Sigma_{1}^{m}$ to $\tilde{C}^{n-2}$ with the following properties:

(i) The projection $\tilde{p}$ sends $\tilde{a}$ homeomorphically onto its image, which we call $a$;

(ii) the arc $a$ intersects the branch set only at one of its endpoints $P$, on $C^{n-2}$, and the ball $\Delta_{1}^{m+1}$ only at its other endpoint $Q$, on $\Sigma^{m}$.

We obtain a framed disc $\Delta^{m}$ from the framed $\Sigma^{m}$ by running a tube from $\Sigma^{m}$ to $C^{n-2}$ along $a$ (see $\$ 6$ ). By Lemma 4 one of the components of the inverse image of the framed $\Delta^{m}$ is a framed symmetric $m$-sphere $\Sigma_{1, h+1}^{m}$ which is the result of running a tube from the framed $\Sigma_{1}^{m}$ to the framed $\Sigma_{h+1}^{m}$.

Since the framed sphere $\Sigma_{h+1}^{m}$ bounds a disc and its framing extends to it, we can use Lemma 2 to deduce that the framed symmetric $\Sigma_{1, h+1}^{m}$ is ambient isotopic to the framed sphere $\Sigma_{1}^{m}$.

Choosing another one of the $\Sigma_{i}^{m}$, say $\Sigma_{2}^{m}$, we can do this again, First we must shrink the sphere $\Sigma^{m}$ so that the image of the shrinking does not intersect a regular neighborhood of $\Sigma^{m}$. By general position, since $m<n-2$, we can choose an arc $\tilde{a}_{2}$ so that $\tilde{p} \tilde{a}_{2}$ does not intersect $a$, and now we can repeat the entire process. This proves Theorem 1 .

\section{Applications.}

THEOREM 2. Let $p: M^{n} \rightarrow S^{n}$ be an $h$ to one-branched covering. For any $m>0$ there is an $h+1$ to one-branched covering $\hat{p}: M^{n} \times S^{m} \rightarrow S^{n+m}$. If $B^{n-2} \subset S^{n}$ is the branch set for $p$ then the branch set for $\hat{p}$ is homeomorphic to the disjoint union $B^{n-2} \times S^{m} \cup B^{m+n-2}$ where $B^{m+n-2}$ is obtained by a sequence of $h$ surgeries of codimension $m$ on $a(m+n-2)$-sphere. Moreover, when $m=2$, these surgeries are performed along unknotted spheres. In particular, if $B^{n-2}$ is orientable so is the new branch set, and if $p$ is a simple branched covering so is $\hat{p}$.

Proof. Let $i: D^{n} \rightarrow S^{n}$ be the inclusion of a ball away from the branch set and let $\alpha: D^{n} \times S^{m} \rightarrow S^{n} \times S^{m}$ be the embedding $i \times 1$. Let $\tilde{\alpha}_{1}, \ldots, \tilde{\alpha}_{h}$ be the embeddings of $D^{n} \times S^{m} \rightarrow M^{n} \times S^{m}$ obtained by the $h$ different lifts of $\alpha$ in the branched covering $p \times 1: M^{n} \times S^{m} \rightarrow S^{n} \times S^{m}$. We do surgeries of type $(m+$ $1, n)$ on $\alpha$ and $\tilde{\alpha}_{1}, \ldots, \tilde{\alpha}_{h}$ simultaneously. This gives rise to a branched covering $\tilde{p}: \tilde{M}^{n+m} \rightarrow S^{n+m}$ where $\tilde{M}^{n+m}$ is obtained from $M^{n} \times S^{m}$ by the $h$ surgeries on the $\tilde{\alpha}_{i}\left(D^{n} \times S^{m}\right)$, and $S^{n+m}$ is obtained from $S^{n} \times S^{m}$ by the surgery on $\alpha\left(D^{n} \times S^{m}\right)$.

Let $\Delta^{n}$ be $S^{n}-$ int $i\left(D^{n}\right)$ and let $k: D^{m} \rightarrow S^{m}$ be an embedding. Then $\Delta^{n} \times D^{m} \stackrel{\left.\text { (id } x^{k}\right)}{\rightarrow}\left(S^{n}-\right.$ int $\left.i\left(D^{n}\right)\right) \times S^{m} \rightarrow S^{n+m}$ defines an embedding $\beta^{\prime}$. Restricting $\beta^{\prime}$ to $\left(\Delta^{n}-\right.$ int $\left.\Delta_{0}^{n}\right) \times D^{m}$ we get an embedding $\beta$ : $S^{n-1} \times I \times D^{m} \rightarrow S^{n+m}$ where $\Delta_{0}^{n} \subset$ Int $\Delta^{n}$ has been chosen large enough so that $\Delta^{n}-$ int $\Delta_{0}^{n}$ does not intersect the branched set. 
Let $\tilde{\beta}_{1}, \ldots, \tilde{\beta}_{h}: S^{n-1} \times I \times D^{m} \rightarrow \tilde{M}^{n+m}$ be the $h$ distinct lifts of $\beta$, indexed so that image $\tilde{\beta}_{j}$ intersects image $\tilde{\alpha}_{j}$ for $1<j \leqslant h$.

Since $\beta\left(S^{n-1} \times 0 \times\{0\}\right)$ is disjoint from the branch set and bounds an $n$-disc in $S^{n+m}$, Theorem 1 and Lemma 3 show that we may alter the $h$ to one-branched covering $\tilde{p}: \tilde{M}^{n+m} \rightarrow S^{n+m}$ to obtain the $h+1$ to one-branched covering $\hat{p}$ : $\chi \tilde{M}^{n+m} \rightarrow S^{n+m}$ where $\chi \tilde{M}^{n+m}$ is obtained from $\tilde{M}^{n+m}$ by surgeries of type $(n, m+1)$ on the embeddings $\tilde{\beta}_{1}, \ldots, \tilde{\beta}_{h}$. The surgery of type $(m+1, n)$ on $\tilde{\alpha}_{j}$ followed by the surgery of type $(n, m+1)$ on $\tilde{\beta}_{j}$ has no effect on the topological type of $M^{n} \times S^{n}$ at all because of the particular definition of the embedding $\beta$. Thus $\chi \tilde{M}^{n+m}=M^{n} \times S^{m}$. The truth of the statements about the branch set and simplicity of the coverings follows easily from Theorem 1, Lemma 3 and the construction.

COROLlARY 2. The manifold $S^{n_{1}} \times \cdots S^{n_{k}}$ is a simple $k$-fold branched covering of $S^{n_{1}+\cdots+n_{k}}$ branched over an orientable, codimension 2 , locally flat manifold.

In particular we note that the $\boldsymbol{n}$-dimensional torus is an $\boldsymbol{n}$-fold branched covering space of $S^{n}$.

TheORem 3. Let $p: M^{n} \rightarrow S^{n}$ be an $h$ to one-branched covering space and let $f$ : $M^{n} \rightarrow M^{n}$ be a fibre preserving homeomorphism that is the identity on the ball $B^{n}$. Then

(a) the "mapping torus" of $f\left(M^{n} \times I /(x, 0)=(f(x), 1)\right)$, and

(b) the "open book" with leaf $M^{n}$-interior $B^{n}$ and monodromy $f \mid\left(M^{n}\right.$-interior $\left.B^{n}\right)$ are both $h+1$ to one-branched covering spaces of $S^{n+1}$.

If the branch set of $p: M^{n} \rightarrow S^{n}$ was an orientable manifold so is the new branch set.

Proof. We can assume by restricting to a smaller ball, that the ball $p\left(B^{n}\right)=\hat{B}^{n}$ is disjoint from the branch set. The "mapping torus" of $f$ is an $h$ to one-branched covering space of the "mapping torus" of $\hat{f}=p f p^{-1}$. This manifold is $\left(S^{n} \times I /(x, 0)=(\hat{f}(x), 1)\right)$ which is homeomorphic to $S^{n} \times S^{1}$, via a homeomorphism fixing $\hat{B}^{n} \times I /(x, 0)=(x, 1)$ because $\hat{f}$ is isotopic to the identity using the Alexander trick. We can do type $(2, n)$-surgery on $\hat{B}^{n} \times S^{1}$ and each of its $h$ lifts to obtain the $h$ to one-branched covering $q: X^{n+1} \rightarrow S^{n+1}$, where $X^{n+1}$ is a manifold obtained from the mapping torus of $f$ by $h$ surgeries. Now we can add a trivial sheet and use Theorem 1 and Lemma 3 to kill all $h$ of the surgeries, proving part (a), or to kill only $h-1$ of the surgeries, proving part (b).

Appendix: The framing restriction. In this appendix we will work in the differential category.

Let $\Sigma^{k}$ be a symmetric $k$-sphere with respect to $p: M^{n} \rightarrow\left(N^{n}, B^{n-2}\right)$ as at the beginning of $\$ 5$.

The different ways of attaching a $(k+1)$-handle to $M^{n} \times I^{1}$ along the tube $\hat{U}=\hat{\psi}\left(S^{k} \times D^{n-k}\right) \times\{1\}$ correspond to the elements of $\pi_{k}(S O(n-k))$. In fact, given a function $f: S^{k} \rightarrow S O(n-k)$ we obtain a new embedding $\hat{\psi} \varphi: S^{k} \times D^{n-k}$ $\rightarrow M^{n}$, where $\varphi: S^{k} \times D^{n-k} \rightarrow S^{k} \times D^{n-k}$ is the homeomorphism defined by 
$\varphi(x, y)=(x, f(x) y)$. In order that $\hat{\psi} \varphi$ be symmetric with respect to $p$ (i.e. be the lifting of some $\left.\psi^{\prime}:\left(D_{-}^{n}, \partial E^{k} \times E^{n-k-1}\right) \rightarrow\left(N^{n}, B^{n-2}\right)\right)$ it is necessary and sufficient that $\varphi$ commutes with the involution $\left(\rho_{k+1} \mid S^{k}\right) \times \rho_{n-k}$ defining the covering $q_{1}$, or equivalently, that $f\left(\rho_{k+1} x\right)=\rho_{n-k} f(x) \rho_{n-k}$, for each $x \in S^{k}$, where $\rho_{n}$ : $D^{n} \rightarrow D^{n}$ denotes the reflection through $D^{n-1} \subset D^{n}$.

The group of such functions (modulo homotopies such that $f_{t}\left(\rho_{k+1} x\right)=\rho_{n-k}$ $f_{t}(x) \rho_{n-k}$, for each $\left.t \in I, x \in S^{k}\right)$ is isomorphic to $\pi_{k}(S O(n-k), O(n-k-1))$. To see this, note that such a function is completely determined by its values on $D_{+}^{k}$, and note that $f(x)$ preserves $D^{n-k-1}$ if $x \in \partial D_{+}^{k}$.

There is a map $R: \pi_{k}\left(S O(n-k), O(n-k-1) \rightarrow \pi_{k}(S O(n-k))\right)$, defined as follows: If $\alpha:\left(D_{+}^{k}, S^{k-1}\right) \rightarrow(S O(n-k), O(n-k-1))$, then $R(\alpha)=\alpha$ on $D_{+}^{k}$ and $R(\alpha)=\rho_{n-k} \alpha \rho_{k+1}$ in $D_{-}^{k}$. Thus all the framings of $\Sigma^{k}$ can be made symmetric if and only if $R$ is surjective. One can check that $R$ is surjective if $k=1,2$. If $k>2$, then $\pi_{k}(S O(n-k), O(n-k-1)) \approx \pi_{k}(S O(n-k), S O(n-k-1)) \approx$ $\pi_{k}\left(S^{n-k-1}\right)$ and it becomes a difficult problem to determine when we can change the sewing map to suit our convenience. For example, for $(k, n)=(3,7),(3,8)$, $(4,8), R$ is not surjective.

\section{REFERENCES}

1. A. Edmonds, Extending a branched covering over a handle (preprint).

2. I. Berstein and A. Edmonds, On the construction of branched coverings of low-dimensional manifolds, Trans. Amer. Math. Soc. 247 (1979), 87-124.

3. The degree and branch set of a branched covering, Invent. Math. (to appear).

4. J. Montesinos, Sobre la conjetura de Poincaré y los recubridores ramificados sobre un mudo, Thesis, Universidad Complutense, Madrid, Spain, 1971.

5. Three-manifolds as 3-fold branched covers of $S^{3}$, Quart. J. Math. Oxford Ser. (2) 27 (1976), 85-94.

6. 4-manifolds, 3-fold branched covering spaces and ribbons, Trans. Amer. Math. Soc. 245 (1978), 453-467.

Department of Mathematics, University of Hawail, Honolulu, Hawall 96822 (Curtent address of H. M. Hilden)

School of Mathematics, Institute for Advanced Study, Princeton, New Jersey 08540

Current address (J. M. Montesinos): Facultad de Ciencias, Universidad de Zaragoza, Zaragoza, Spain 